\title{
Synthesis and characterization of organic-inorganic hybrid microspheres
}

\author{
Beata Podkościelna ${ }^{1} \cdot$ Magdalena Sobiesiak $^{1}$
}

Received: 19 October 2015/Revised: 15 November 2015/Accepted: 8 December 2015/Published online: 29 December 2015

(C) The Author(s) 2015. This article is published with open access at Springerlink.com

\begin{abstract}
This study presents the synthesis of hybrid microspheres based on monomers of various chemical nature. As organic compounds, styrene and 1,4-divinylbenzene were used, whereas triethoxyvinylsilane (TEVS) was applied as an inorganic one. Four different systems with the increasing quantity of TEVS were studied. Then inorganic parts of TEVS were removed with aqueous solution of $\mathrm{NaOH}$. Structure of the new materials was confirmed by FTIR-ATR, EDX-SEM and TGA analyses. Thermal resistance of the copolymers was assessed using the TG-DTG system. The synthesized hybrid materials exhibited highly developed porous structure that was confirmed from the low-temperature nitrogen adsorption data. The prepared materials were tested as sorbents for removing organic compounds from aqueous solutions using the SPE technique.
\end{abstract}

Keywords Styrene copolymers - Hybrid microspheres . Triethoxyvinylsilane $\cdot$ Thermal properties $\cdot$ SPE

\section{Introduction}

Hybrid materials are composites generally consisting of two components (inorganic and organic in nature) at the nanometer or molecular size level. This leads to preparation of more homogeneous materials in comparison with traditional compositions.

Magdalena Sobiesiak

magdalena.sobiesiak@umcs.pl

1 Department of Polymer Chemistry, Faculty of Chemistry, Maria Curie-Skłodowska University, M. Curie-Skłodowska sq.5, 20-031 Lublin, Poland
A wide variety of organic-inorganic systems enable to synthesize hybrid materials using different types of synthetic processes.

The organic-inorganic systems provide a wide range of possibilities of synthetic processes for preparation of hybrid materials. Their development is very rapid because they can offer new or significantly better properties of the obtained materials (Agudelo et al. 2011; Liu et al. 2003). The ability to use different methods of synthesis of hybrid materials in the reactions of organic and inorganic reactive monomers makes it possible to directly regulate the properties of the obtained materials (Lebedev 2010).

As an inorganic component silica (silicon (IV) oxide) and its organic derivatives e.g. meth/ethoxysilanes or vinyl silanes are widely used (Grabicka and Jaroniec 2010, Sangermano et al. 2011, Pinto et al. 2012). Silica has a well-developed surface area (up to several hundred $\mathrm{m}^{2} / \mathrm{g}$ ) and a porous structure, which is of great importance in the process of adsorption and ion exchange. Silica and its organic derivatives are widely used in analytical chemistry as packing of chromatographic columns. The modified silica can be also used as sorbents for separation and preconcentration of organic compounds and heavy metal ions from aqueous solution in the solid phase extraction (SPE) technique (Rykowska et al. 2008, 2011, De Conto et al. 2014).

Great interest in silica modified with vinyl groups is recently observed. As a result of polymerization reaction of vinyl groups, silica is chemically built into the structure of the resulting polymer (Donescu et al. 2007, 2013; Agudelo et al. 2011).

Gatica et al. (2003) used vinylsilane monomers (vinyltrimethoxysilane and vinyltriethoxysilane) in a copolymerization reaction with other vinyl monomers: methyl methacrylate (MMA), tert-butyl acrylate (tBA) and 
$\mathrm{N}$-vinyl-2-pyrrolidone (VP) in order to obtain functionalized vinyl copolymers.

Homopolymerization and copolymerization of different alkoxyvinylsilanes with styrene were performed under various conditions in order to select efficient initiating systems for the radical polymerizations (Ihara et al. 2008).

The most frequently used technology for the preparation of hybrid systems is the sol-gel synthesis (Yen and Kuo 2012; Li et al. 2008). The hydrolyzed product of a mixture of tetraethoxysilane and vinyltriethoxysilane was used to produce organic-inorganic hybrid silica by sol-gel processing. The hybrid silica particles were subsequently encapsulated with a layer of PS-DVB, a polymeric mixture of styrene and divinylbenzene. (Li et al. 2003).

Our previously reported microspheres based on methacrylic bisphenols enabled the preparation of modified polymer microspheres with a specific surface area ranging from 30 to $150 \mathrm{~m}^{2} / \mathrm{g}$ (Podkościelna and Kołodyńska 2013; Podkościelna 2011, 2013). This paper focuses on the synthesis of new materials with yet higher porosity. For this purpose triethoxyvinylsilane (TEVS) was used. TEVS is used both for the synthesis and surface modification of various types of sorbents (Chong et al. 2004, Wang et al. 2014).

In this paper synthesis, structure and properties of hybrid microspheres based on styrene (St) 1,4-divinylbenzene (DVB) and triethoxyvinylsilane (TEVS) are presented. In order to obtain a well-developed porous structure, silane part was conveniantly removed. Due to the fact that the TEVS is covalently bonded to the polymeric matrix and triethoxysilane part is removed in the second step, this type of synthesis can be classified as the hard template method. This method is successfully used for preparation of ordered mesoporous carbons (Jaroniec et al. 2009, Peng and Fu 2015, Zhu et al. 2015, Sobiesiak 2012). We used the hard template technique in an innovative way for the preparation of porous polymer microspheres.

To evaluate the efficiency of the used method, characteristic of porosity of the products, before and after removing the silica component was measured. Given the fact that porosity is an important element in the sorption process, studies on the synthesis of porous materials are of considerable importance. The prepared materials will be used as highly porous polymeric adsorbents for SPE organic compounds e.g. phenols.

\section{Experimental}

\subsection{Chemicals and eluents}

St, TEVS, decan-1-ol and bis(2-ethylhexyl)sulfosuccinate sodium salt (DAC,BP) were from Fluka AG (Buchs
Switzerland). $\alpha, \alpha^{\prime}$-Azoiso-bis-butyronitrile (AIBN) and DVB $(62.2 \%$ of 1,4-divinylbenzene, $0.2 \%$ of 1,2-divinylbenzene, and ethylvinylbenzene) washed with $3 \%$ aqueous sodium hydroxide solution before the use were obtained from Merck (Darmstadt, Germany). Acetone, methanol, chlorobenzene, chloroform, hexane, toluene, tetrahydrofuran (THF), acetonitrile and 1,4-dioxane were from POCh (Gliwice, Poland).

\subsection{Synthesis of microspheres}

Copolymerisation of divinylbenzene, styrene and triethoxyvinylsilane was performed in the aqueous medium. $150 \mathrm{~mL}$ of redistilled water and $1.5 \mathrm{~g}$ of bis(2-ethylhexyl)sulfosuccinate sodium salt (surfactant) were stirred for $0.5 \mathrm{~h}$ at $80^{\circ} \mathrm{C}$ in a three-necked flask fitted with a stirrer, a water condenser and a thermometer. Then the solutions containing $10.4 \mathrm{~g}$ of DVB and a corresponding amounts of St and TEVS (Table 1), the initiator AIBN (1 wt \%) and the mixture of pore-forming diluents (toluene and 1-decanol, taken in $1 / 1(\mathrm{v} / \mathrm{v})$ proportions) were added while stirring to the aqueous medium. The reaction mixture was stirred at $350 \mathrm{rpm}$ for $18 \mathrm{~h}$ at $80{ }^{\circ} \mathrm{C}$. The obtained microspheres were washed with distilled hot water (2L), filtered off, dried and extracted in a Soxhlet apparatus with boiling acetone. After drying, the microspheres were fractionated with sieves. The applied polymerisation conditions yielded about $80 \%$ microspheres in the range $50-80 \mu \mathrm{m}$.

\subsection{Procedure to remove silane part}

In a two-necked flask $\left(250 \mathrm{~cm}^{3}\right)$ fitted with a stirrer and a thermometer, $10 \mathrm{~g}$ of copolymers (DVB-St-TEVS) and $50 \mathrm{~mL}$ acetone were added and slowly stirred for $2 \mathrm{~h}$. Next, acetone was filtered and sodium hydroxide solution (150 $\left.\mathrm{mL} \mathrm{H}_{2} \mathrm{O}+9 \mathrm{~g} \mathrm{NaOH}\right)$ was added. The whole content was stirred for $1 \mathrm{~h}$ at room temperature, then for $4 \mathrm{~h}$ at $100{ }^{\circ} \mathrm{C}$ and finally for $15 \mathrm{~h}$ at room temperature. Next, microspheres were filtered off and transferred to a glass beaker and the aqueous solution of $\mathrm{HCl}(200 \mathrm{~mL}$ $\mathrm{H}_{2} \mathrm{O}+10 \mathrm{~mL} \mathrm{HCl}$ ) was added. In the end the mixture was filtered off and dried in a heating oven.

Due to the use basic conditions during the removing of triethoxysilane part, the most probable course of reaction is the hydrolysis of ethoxyl groups to alcohols and sodium silicate.

\subsection{Polymers characterization}

Attenuated Total Reflectance spectroscopy (ATR-FTIR) spectra were recorded on a Bruker TENSOR 27 apparatus (Germany), equipped with diamond crystal. The spectra were gathered from 600 to $4000 \mathrm{~cm}^{-1}$. 
Table 1 Experimental parameters of the synthesis

\begin{tabular}{|c|c|c|c|c|c|c|c|c|}
\hline \multirow[t]{2}{*}{ Copolymers } & \multicolumn{3}{|c|}{ Monomers (g) } & \multicolumn{3}{|c|}{ Molar ratio } & \multirow{2}{*}{$\begin{array}{l}\text { Initiator }(\mathrm{g}) \\
\text { AIBN }\end{array}$} & \multirow[t]{2}{*}{ Product } \\
\hline & DVB & St & TEVS & DVB & St & TEVS & & \\
\hline DVB-ST & 10.4 & 8 & 0 & 1 & 1 & 0 & 0.184 & M \\
\hline DVB-ST-TEVS 0.25 & 10.4 & 6.24 & 3.8 & 1 & 0.75 & 0.25 & 0.204 & M \\
\hline DVB-ST-TEVS 0.5 & 10.4 & 4.16 & 7.6 & 1 & 0.5 & 0.5 & 0.221 & M \\
\hline DVB-ST-TEVS 0.75 & 10.4 & 2.08 & 11.4 & 1 & 0.25 & 0.75 & 0.238 & $\mathrm{C}$ \\
\hline DVB-TEVS & 10.4 & 0 & 15.2 & 1 & 0 & 1 & 0.256 & $\mathrm{C}$ \\
\hline
\end{tabular}

$M$ Microspheres, $C$ Coagulation
Microanalysis of elements was carried out using the scanning electron microscope EDX SEM (FEI company), equipped with the detector type: SDD Apollo XL, resolution: 132.14 , Lsec: 50.

Thermogravimetric analyses (TG/DTG) were performed with a thermal analyzer STA 449 F1 Jupiter (Netzsch, Selb, Germany). $\mathrm{Al}_{2} \mathrm{O}_{3}$ pans were used for measurements. The sample weight was about $10 \mathrm{mg}$. Dynamic scans were performed at the heating rate of $10 \mathrm{~K} / \mathrm{min}$ in the temperature range $30-1000{ }^{\circ} \mathrm{C}$, under synthetic air atmosphere (40 $\mathrm{mL} / \mathrm{min})$. The initial decomposition temperature $\left(T_{\mathrm{ini}}\right)$, $T_{10} \%, T_{50} \%$ of weight loss and that of the maximum rate of weight loss $\left(T_{\max 1}\right.$ and $\left.T_{\max 2}\right)$ were determined.

The swellability coefficient $B$ was determined by equilibrium swelling in acetone, methanol, propan-2-ol, dichloromethane, acetonitryle, chloroform, toluene, hexane THF and 1,4-dioxane using the method proposed by Tuncel and Pişkin 1996, where $B$ is expressed as:

$B=\frac{V_{\mathrm{s}}-V_{\mathrm{d}}}{V_{\mathrm{d}}} \times 100 \%$

where: $V_{\mathrm{s}}$ is the volume of the copolymer after swelling and $V_{\mathrm{d}}$ is the volume of the dry copolymer.

The polymer sample $(0.5 \mathrm{~g})$ was placed into the cylindrical glass tube with porous ceramic frit and put in the glass vessel containing c.a. $2 \mathrm{~mL}$ of solvent. The volume of polymer samples was measured before and after experiment.

The porous structure of copolymers was characterized by nitrogen adsorption at $77 \mathrm{~K}$ using an adsorption analyzer ASAP 2405 (Micrometrics Inc., USA). Before analysis the copolymers were outgassed at $150{ }^{\circ} \mathrm{C}$ for $2 \mathrm{~h}$. Specific surface areas were calculated by the BET method, assuming that the area of a single nitrogen molecule in the adsorbed state is $16.2 \AA^{2}$. Total pore volumes were calculated from single point adsorption. Pore size distributions were determined by the BJH method.

\subsection{Solid phase extraction (SPE) experiments}

The off-line SPE method was used for the estimation of sorption capabilities of hybrid materials towards phenol and its chlorinated derivatives. The mixture of phenols was preconcentrated from aqueous solution using laboratory cartridges filled with $100 \mathrm{mg}$ of the studied material. The aqueous solutions of phenols were prepared by dilution $(1: 50 \mathrm{v} / \mathrm{v})$ of the standard methanolic solution containing $100 \mathrm{mg} / \mathrm{L}$ of: phenol (P), 2-chlorophenol (2-ChP), 2,4dichlorophenol (2,4-DChP), and 2,4,6-trichlorophenol (2,4,6-TChP). The increasing sample volumes of the aqueous solutions were sucked through the cartridge using the water aspirator. Then the phenolic compounds were eluted with methanol ( $2 \mathrm{~mL}$ of methanol for each $100 \mathrm{~mL}$ of sucked sample volume). Concentration of phenols in the obtained eluates was determined with the HPLC method. The details of the analysis were described in our previous papers (Sobiesiak and Podkościelna 2010; Sobiesiak 2011; Gawdzik et al. 2005). Recovery values were calculated as the mean of three measurements. Decrease of the recovery below $25 \%$ of the maximum value was assumed as a breakthrough volume for the compound under consideration.

\section{Results and discussion}

This paper presents a new way of synthesis of porous materials, consisting of divinylbenzene (crosslinker), styrene and triethoxyvinylsilane (TEVS). Due to presence of vinyl bonds in TEVS structure, this compound is able to react with St and DVB. Therefore, the silane part is chemically bonded with organic part and not only physically mixed as in case of commonly used mineral silica. Moreover, the proposed method allow to prepare the hybrid material in spherical shape with highly developed porous structure.

The chemical structures of monomers used in the suspension-emulsion copolymerization are presented in Fig. 1 . An attempt to synthesize four different systems with the increasing quantity of TEVS was made (Table 1). The syntheses of microspheres were successful for the systems with the TEVS content up to $0.5 \mathrm{~mol}$. For higher values of TEVS, the coagulation process took place. 


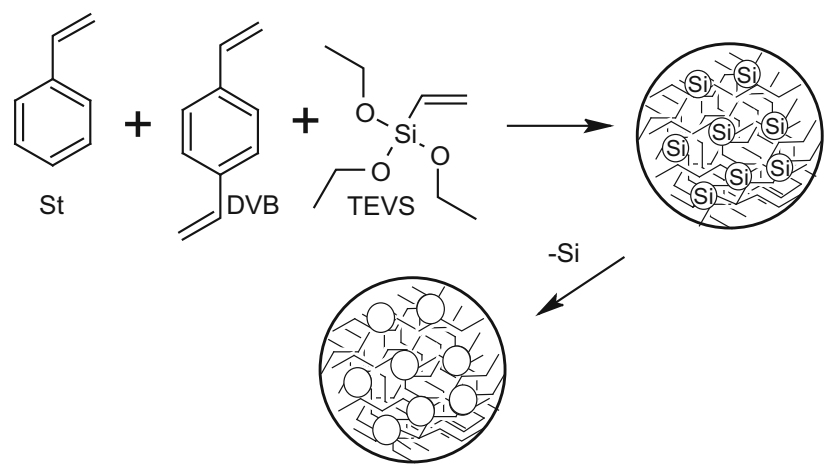

Fig. 1 Chemical structure of monomers

In order to prove that TEVS was incorporated into the polymeric structure and subsequently silane part was removed, FTIR-ATR spectra and EDAX-SEM analyses were performed.

Figure 2 presents the infrared spectra, before (DVB-STTEVS 0.5) and after (DVB-ST 0.5) removing of silane part of TEVS. In the spectra, one can see a very strong band at $1000-1110 \mathrm{~cm}^{-1}$ which corresponds to the asymmetrical stretching vibration of $\mathrm{Si}-\mathrm{O}-\mathrm{C}$ group. The presence of this signal confirms the incorporation of TEVS into the polymeric structure of the synthesized material. After removing silane from the polymeric network, this band is absent in the spectra which proves the correct course of the process. Moreover, the spectrum of the DVB-ST 0.5 looks almost the same as for the DVB-ST, which was prepared without the addition of TEVS.

Elemental analysis carried out using the scanning electron microscope (EDX-SEM) shows the presence or lack of $\mathrm{Si}$ atoms on the microspheres surface. The microanalysis data with a marked percentage of silicon (wt $\%$ ) are shown in Fig. 3. These measurements confirm the presence or disappearance of silicon in the polymers, as well. The Si content may be undervalued because the measurement is made only on the surface of the microspheres.

Thermal analysis TG-DTG also verifies the above described results. The values of residual mass after thermal analysis are in agreement with the EDX-SEM data. The results of TG-DTG measurements are shown in Table 2 and Fig. 4. The initial decomposition temperature $\left(T_{\mathrm{ini}}\right)$ for all copolymers is in the range $265-272{ }^{\circ} \mathrm{C}$ and the final decomposition process takes place at ca. $600{ }^{\circ} \mathrm{C}$. The decomposition process occurs in two stages: first at ca $330{ }^{\circ} \mathrm{C}$ and second ca $500{ }^{\circ} \mathrm{C}$. In the first stage, decomposition of polymeric aliphatic parts and formation of carbonaceous structure take place while in the second step, decomposition of aromatic and carbon structures is observed. After removing silicon part, slight increase of copolymers thermal stability is noted.

Swellability is a factor that defines accessibility of internal chemical structure in crosslinked polymers for penetration by solvent molecules. The results of the swellability coefficient $(B)$ for the studied copolymers are collected in Table 3. The values of $(B)$ for all microspheres vary from 0 to $45 \%$. The largest values are observed for the DVB-ST in THF (45\%), toluene (40\%) and methanol (33\%). Significantly lower values are found for copolymers with TEVS, which vary from 0 to $22 \%$. The copolymers possessing silane part in their structure show lower tendency to swell contrary to those after removal of inorganic part. It means that silane part increases the rigidity of the polymer networks and prevents penetration of the diluents.
Fig. 2 FTIR-ATR spectra of the studied materials

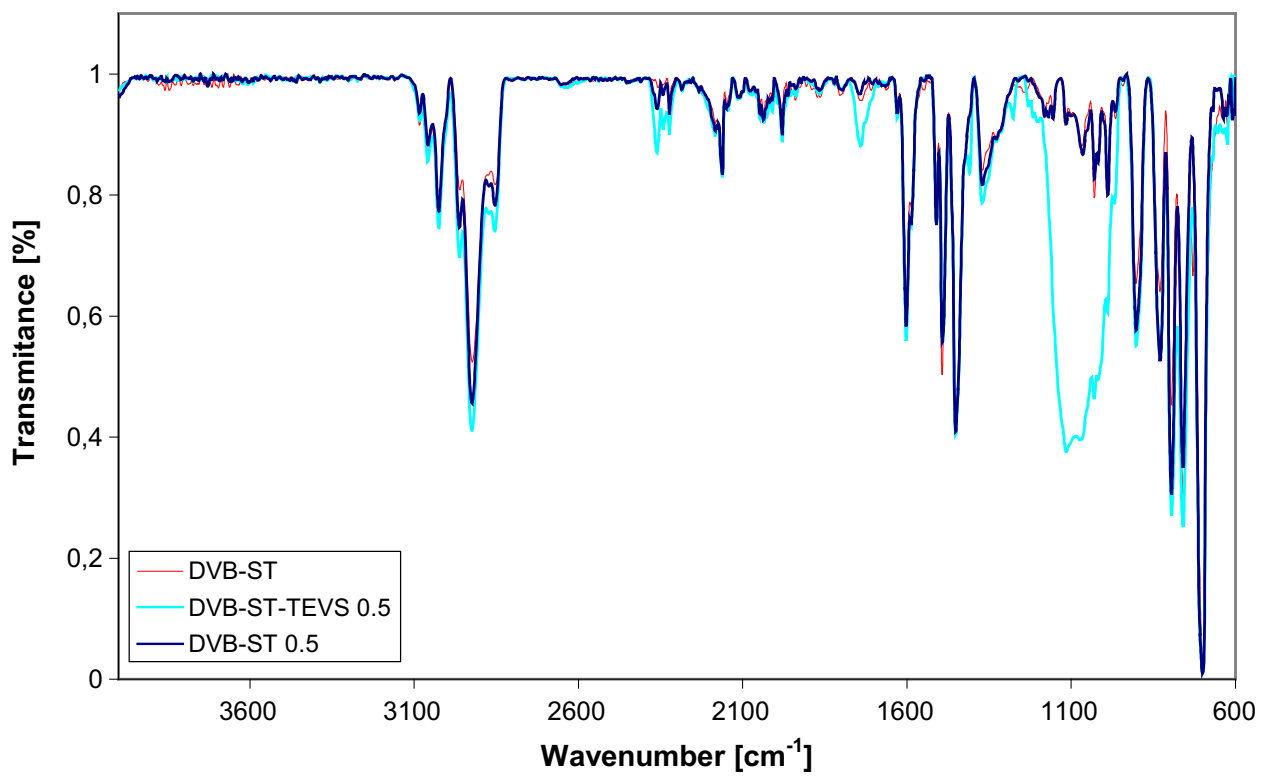



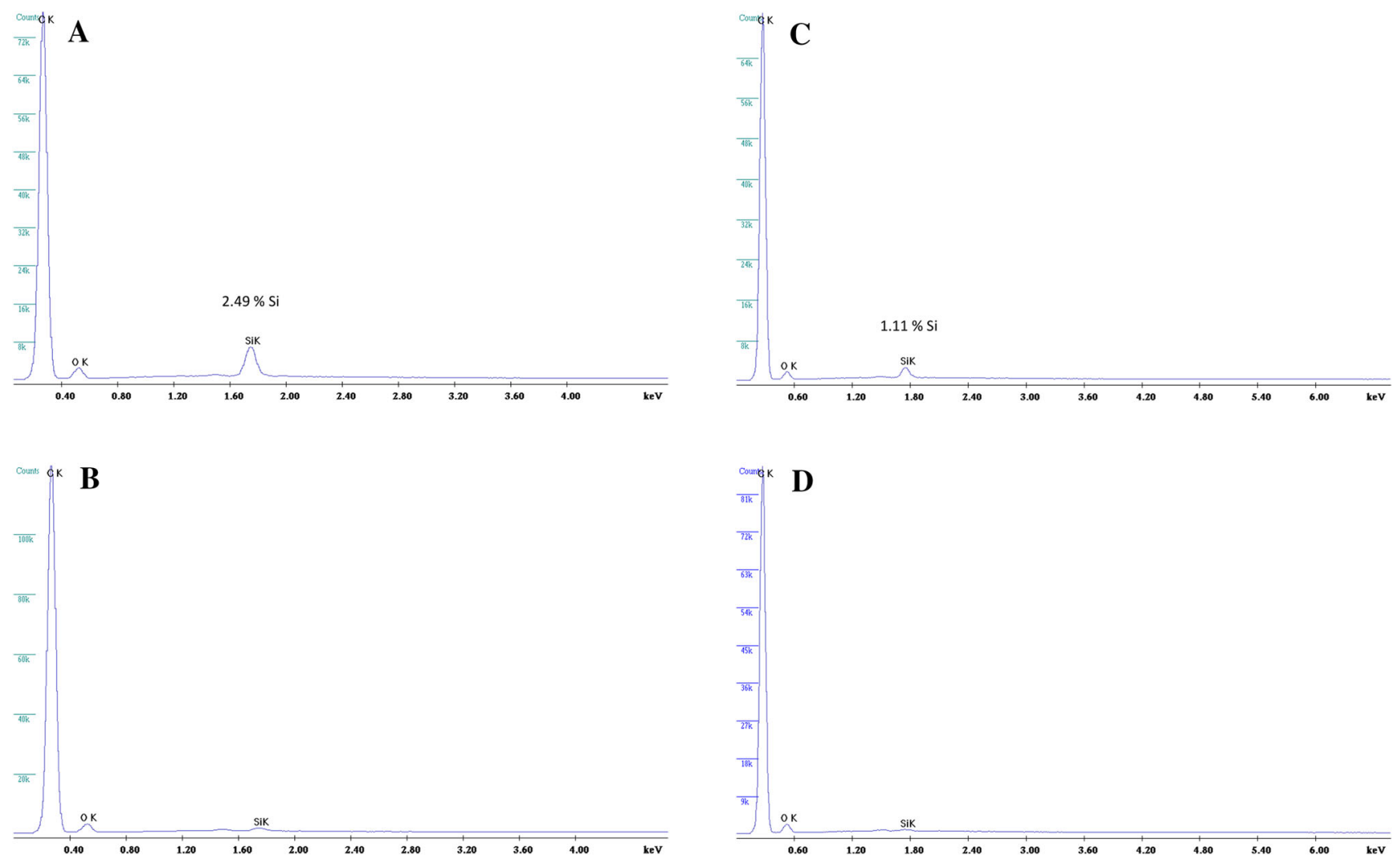

Fig. 3 Microanalysis report (a, b) DVB-St-TEVS (1:0.5:0.5) A-with TEVS, B-after remove TEVS (c, d) DVB-St-TEVS (1:0.75:0.25) C-with TEVS, D-after remove TEVS

Table 2 Thermal properties of copolymers

\begin{tabular}{lllllll}
\hline Copolymer & \multicolumn{2}{l}{ Temperature $\left({ }^{\circ} \mathrm{C}\right)$} & & $m_{\mathrm{r}}(\%)$ \\
\cline { 2 - 6 } & $T_{\text {ini }}$ & $T_{10 \%}$ & $T_{50 \%}$ & $T_{\max 1}$ & $T_{\max 2}$ & \\
\hline DVB-ST & 265.0 & 293.8 & 412.6 & 327.8 & 527.3 & 1.38 \\
DVB-ST-TEVS 0.25 & 268.7 & 308.4 & 399.1 & 328.2 & 501.5 & 1.85 \\
DVB-ST 0.25 & 272.2 & 311.6 & 393.6 & 331.6 & 510.8 & 1.16 \\
DVB-ST-TEVS 0.5 & 267.0 & 306.6 & 420.1 & 324.2 & 475.2 & 5.87 \\
DVB-ST 0.5 & 270.1 & 314.7 & 389.4 & 342.1 & 496.7 & 1.45 \\
\hline
\end{tabular}

$T_{\text {ini }}$ initial decomposition temperature, $T_{10} \% T_{50} \%$ of the rate of weight loss, $T_{\max 1,2}$ the maximum of decomposition temperature at first or second stage, $m_{r}$ residual mass
Table 4 presents brief characteristics of porous structure parameters obtained for the prepared materials. Specific surface areas have high values for polymeric materials and they are in the range 230 to $\mathrm{ca} 400 \mathrm{~m}^{2} / \mathrm{g}$. The total pore volume ranges form 0.905 to $1.206 \mathrm{~cm}^{3} / \mathrm{g}$. All the materials are mesoporous and the contents of micropores do not exceed $2 \%$. The copolymers prepared with the addition of TEVS have much higher porosity than DVB-ST. Thus, TEVS has very positive influence on porosity development. After removing silane part, slight increase of porous structure parameters with the decreasing percentage of micropores is observed. It means that removing silane part unblocked some of the pores. This effect can be seen also in Fig. 5, where the pore size distribution curves are shown especially for the St-DVB 0.25 material. The main difference occurs in range of $30-40 \AA$. TEVS is incorporated into the structure of polymeric network only in limited range of concentrations. In consequence one can observe a decrease of reactivity of the whole system with an increase of TEVS amount. 


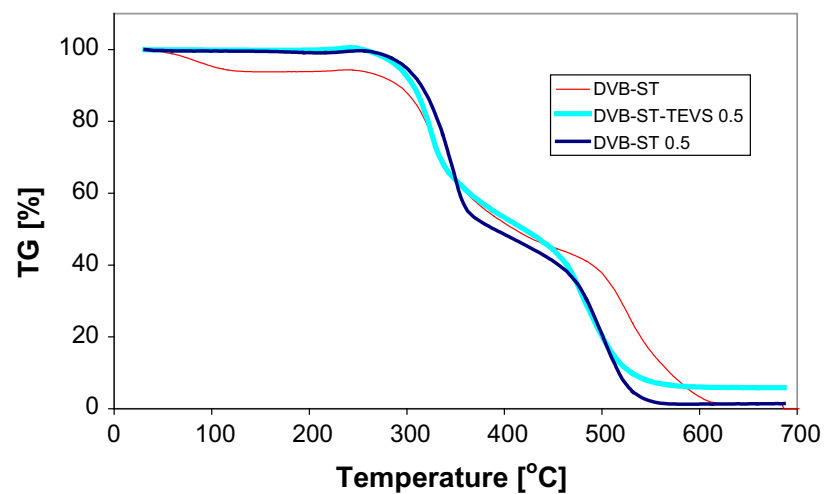

Fig. 4 Results of thermogravimetric analyses for DVB-ST, DVB-STTEVS 0.5 and DVB-ST 0.5

Increase of pore volume in this range should have a positive effect on sorption processes carried out on these materials. This assumption finds confirmation in the results from SPE experiments.

The results of SPE experiments are presented in Fig. 6. Phenols have low affinity to the surface of DVB-ST copolymer. The highest value of recovery was $55 \%$ and it was obtained for TChP. Phenol had the smallest affinity towards this material therefore the maximum recovery for this compound was only ca $5 \%$. The studied hybrid materials possessed much better sorption ability than the DVB-ST. For all these materials maximum recoveries of TChP exceeded $90 \%$ and reached up to $100 \%$. Also for phenol, 4-6 fold increase in the recovery values was observed. Another factor decisive for sorption abilities of a

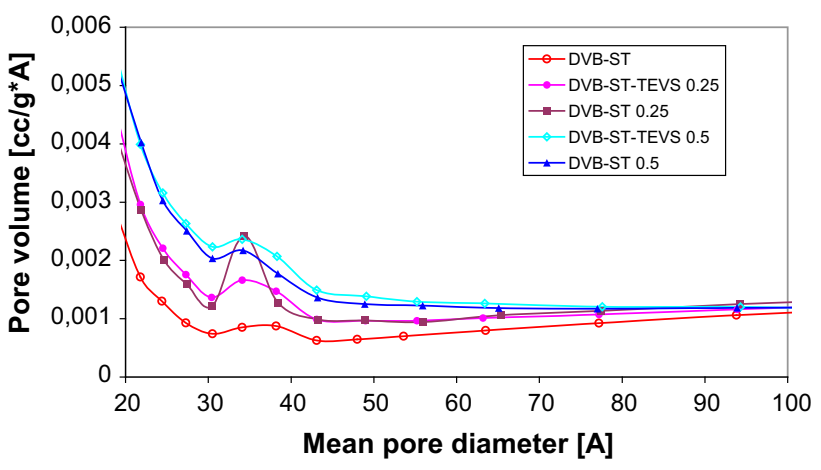

Fig. 5 Pore size distributions of the analyzed materials

sorbent is the breakthrough volume. The higher the breakthrough volume value is, the better sorption capacity the studied material possesses. For the DVB-ST-TEVS 0.25 breakthrough volume is in the range $500-600 \mathrm{~mL}$, but after removal of silane part (DVB-ST 0.25), its values rise up to $800 \mathrm{~mL}$. In the case of DVB-ST-TEVS 0.5 breakthrough of the sorbent bed is rapid and it takes place at the volume of $900 \mathrm{~mL}$. Removal of silane part (DVB-ST 0.5) caused the breakthrough process getting more slowly and the breakthrough volume somehow shifted towards higher values. These observations and comparison of the courses of the pore size distribution curves (Fig. 5) imply that the increase in the pore volume from 30 to $40 \AA$ caused by removal of silane part, had an essential effect on improvement of sorption properties of the studied materials.
Table 3 Swelling studies

Table 4 Porous structure parameters

\begin{tabular}{lllllll}
\hline Copolymer & \multicolumn{7}{l}{ Swellability coefficient, B (\%) } \\
\cline { 2 - 6 } & Acetone & Methanol & THF & Toluene & Acetonitryle & Water \\
\hline DVB-ST & 30 & 33 & 45 & 40 & 24 & 5 \\
DVB-ST-TEVS 0.25 & 11 & 5 & 18 & 10 & 10 & 0 \\
DVB-ST 0.25 & 21 & 9 & 22 & 12 & 15 & 0 \\
DVB-ST-TEVS 0.5 & 17 & 7 & 5 & 2 & 7 & 0 \\
DVB-ST 0.5 & 20 & 10 & 9 & 5 & 9 & 0 \\
\hline
\end{tabular}

\begin{tabular}{llllll}
\hline Copolymers & $S_{\text {BET }}\left(\mathrm{m}^{2} / \mathrm{g}\right)$ & $V_{\text {Tot }}\left(\mathrm{cm}^{3} / \mathrm{g}\right)$ & $V_{\text {Micro }}\left(\mathrm{cm}^{3} / \mathrm{g}\right)$ & $\%_{\text {Micro }}$ & $W(\mathrm{~nm})$ \\
\hline DVB-ST & 230 & 0.905 & - & - & 15.7 \\
DVB-ST-TEVS 0.25 & 343 & 1.020 & 0.007 & 0.68 & 11.8 \\
DVB-ST 0.25 & 349 & 1.080 & 0.003 & 0.28 & 12.4 \\
DVB-ST-TEVS 0.5 & 387 & 1.024 & 0.016 & 1.56 & 10.5 \\
DVB-ST 0.5 & 391 & 1.206 & 0.011 & 0.91 & 12.3 \\
\hline
\end{tabular}



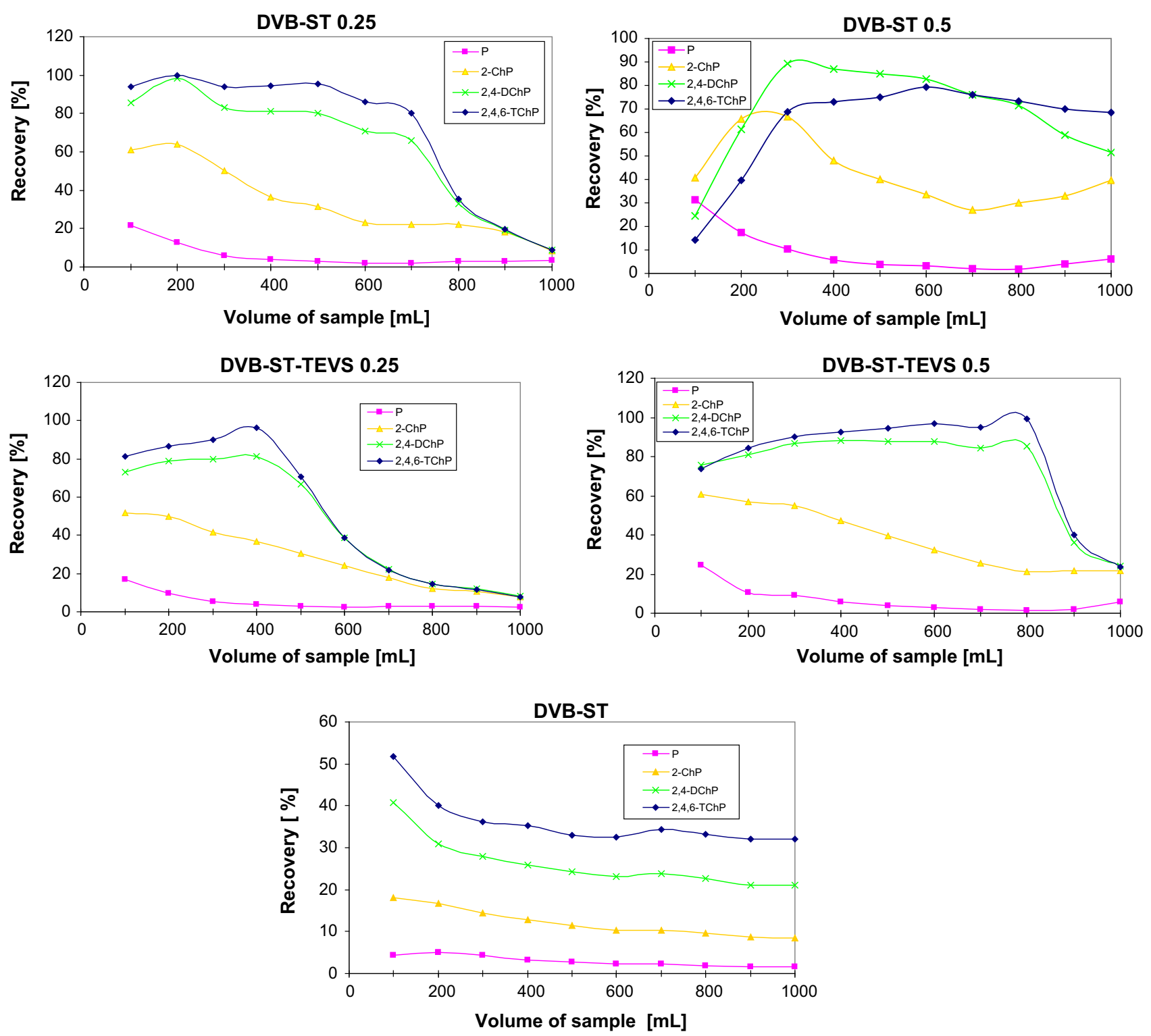

Fig. 6 Results of solid phase extraction of phenols obtained for the presented materials

\section{Conclusions}

The studies presented in this paper show a new method of synthesis of hybrid materials based on styrene, divinylbenzene and triethoxyvinylsilane. The effective method of silane part removal was proposed which was confirmed by FTIR-ATR, EDX-SEM and TGA measurements.

The obtained hybrid copolymers have good thermal resistance, the initial decomposition temperature is in the range $265-272{ }^{\circ} \mathrm{C}$, while the decomposition process is over at ca. $600{ }^{\circ} \mathrm{C}$. The prepared materials possess a well developed porous structure. The presence of pores of the sizes between 30 and $40 \AA$ has positive influence on dynamic sorption of phenols. The SPE experiments proved high efficiency of these materials in both removing phenols from aqueous solution as well as in the desorption processes (recovery up to $100 \%$ ).

Acknowledgments The research leading to these results has received funding from the People Programme (Marie Curie Actions) of the European Union's Seventh Framework Programme FP7/2007-2013/ under REA grant agreement No PIRSES-GA-2013-612484.

Open Access This article is distributed under the terms of the Creative Commons Attribution 4.0 International License (http://crea tivecommons.org/licenses/by/4.0/), which permits unrestricted use, distribution, and reproduction in any medium, provided you give appropriate credit to the original author(s) and the source, provide a link to the Creative Commons license, and indicate if changes were made. 


\section{References}

Agudelo, N.A., Perez, L.D., Lopez, B.L.: A novel method for the synthesis of polystyrene-graft-silica particles using random copolymers based on styrene and triethoxyvinylsilane. Appl. Surf. Sci. 257, 8581-8586 (2011)

Chong, A.S.M., Zhao, X.S., Kustedjo, A.T., Qiao, S.Z.: Functionalization of large-pore mesoporous silicas with organosilanes by direct synthesis. Micropor. Mesopor. Mat. 72, 33-42 (2004)

De Conto, J.F., Santos, M.R.O., Carvalho, A.S., Campos, K.V., Freitas, L.S., Benvenutti, E.V., de Menezes, E.W., Santana, C.C., Egues, S.M.: Naphthenic acids recovery from petroleum using ionic silica based hybrid material as stationary phase in solid phase extraction (SPE) process. Adsorption 20, 917-923 (2014)

Donescu, D., Serban, S., Petcu, C., Nistor, C.L., Ghiurea, M., Corobea, M.C.: Polymer-silica hybrids obtained by microemulsion polymerization. Colloid Polym. Sci. 285, 1455-1462 (2007)

Donescu, D., Somoghi, R., Spataru, C.I., Manaila-Maximean, D., Panaitescu, D.M., Vasile, E., Nistor, C.L.: Hybrid polymeric latexes containing magnetite. Colloid Polym. Sci. 291, 2345-2358 (2013)

Gatica, N., Fernandez, N., Opazo, A., Alegria, S., Gargallo, L., Radic, D.: Synthesis and characterization of functionalized vinyl copolymers. Electronegativity and comonomer reactivity in radical copolymerization. Polym. Int. 52, 1280-1286 (2003)

Gawdzik, B., Sobiesiak, M., Puziy, A.M., Poddubnaya, O.I.: Carbon sorbents derived from porous polymers for off-line preconcentration of chlorophenols from water. J. Liq. Chromatogr. Relat. Technol. 27, 1027-1041 (2005)

Grabicka, B.E., Jaroniec, M.: Adsorption properties of ordered mesoporous silicas synthesized in the presence of block copolymer Pluronic F127 under microwave irradiation. Adsorption 16, 385-396 (2010)

Ihara, E., Kurokawa, A., Itoh, T., Inoue, K.: Homopolymerization and copolymerization with styrene of various alkoxyvinylsilanes and oxidative transformation of $\mathrm{C}-\mathrm{Si}$ bond in the resulting copolymers to afford poly[(vinyl alcohol)-co-styrene]s. Polym. J. 40, 1140-1148 (2008)

Jaroniec, M., Gorka, J., Choma, J., Zawislak, A.: Synthesis and properties of mesoporous carbons with high loadings of inorganic species. Carbon 47, 3034-3040 (2009)

Lebedev, E.V.: Hybrid organo-inorganic polymer systems: synthesis, structure, and properties. Theor. Exp. Chem. 46, 409-415 (2011)

Li, Y.S., Li, B., Han, N.Y., Xu, B.J.: Studies on a poly(styrenedivinylbenzene)-encapsulated sub-micrometer sized organicinorganic hybrid silica packing, its synthesis, some of its characteristics and applications. J. Chromatogr. A 1021, 183-189 (2003)

Li, Li-Jun, Pi, Pi-Hui, Wen, Xiu-Fang, Cheng, Jiang, Yang, Zhuo-Ru: Aluminum pigments encapsulated by inorganic-organic hybrid coatings and their stability in alkaline aqueous media. J. Coat. Technol. Res. 5, 77-83 (2008)
Liu, P., Tian, J., Liu, W.M., Xue, Q.J.: Surface graft polymerization of styrene onto nano-sized silica with a one-pot method. Polym. J. 35, 379-383 (2003)

Peng, X.M., Fu, D.F.: Preparation of ordered mesoporous carbons with ammonia modification for Orange II adsorption, Desalin. Water Treat. 54, 255-264 (2015)

Pinto, M.L., Marques, J., Pires, J.: Porous clay heterostructures with zirconium for the separation of hydrocarbon mixtures. Sep. Pur. Tech. 98, 337-343 (2012)

Podkościelna, B.: Synthesis, modification and porous properties of new glycidyl methacrylate copolymers. J. Appl. Polym. Sci. 120, 3020-3026 (2011)

Podkościelna, B., Kołodyńska, D.: A new type of cation-exchange polymeric microspheres with pendant methylenethiol groups. Polym. Adv. Technol. 24, 866-872 (2013)

Podkościelna, B.: The use of bis[4(2-hydroxy-3-methacryloyloxypropoxy)phenyl]sulfide in preparation of microspheres with pendant amine groups as a heavy metal sorbent. Sep. Sci. Tech. 48, 1699-1708 (2013)

Rykowska, I., Wasiak, W., Szymański, A., Szyrwińska, K., Lulek, J.: Chemically bonded phases for the analysis of trace amounts of organic pollutants. Toxicol. Mech. Meth. 18, 537-542 (2008)

Rykowska, I., Wasiak, w: Chemically modified silica gel for selective solid-phase extraction and preconcentration of heavy metal ions. Int. J. Environ. Anal. Chem. 91, 1466-1476 (2011)

Sangermano, M., El Sayedb, H., Voit, B.: Ethoxysilyl-modified hyperbranched polyesters as mulitfunctional coupling agents for epoxy-silica hybrid coatings. Polymer 52, 2103-2109 (2011)

Sobiesiak, M.: Bead-shaped porous polymers containing bismaleimide-their physico-chemical characteristics and sorption properties towards chlorophenols. Polish J. Appl. Chem. 72, 25-32 (2011)

Sobiesiak, M.: Thermal properties of nanoporous carbons prepared by a template method using different polymeric and organic precursors. New Carbon Mater. 27, 337-343 (2012)

Sobiesiak, M., Podkościelna, B.: Preparation and characterization of porous DVB copolymers and their applicability for adsorption (solid-phase extraction) of phenol compounds. Appl. Surf. Sci. 257, 1222-1227 (2010)

Tuncel, A., Pişkin, A.: Nonswellable and swellable poly(EGDMA) microspheres. J. Appl. Polym. Sci. 62, 789-798 (1996)

Wang, X., Kang, Q., Shen, D., Zhang, Z., Li, J., Chen, L.: Novel monodisperse molecularly imprinted shell for estradiol based on surface imprinted hollow vinyl- $\mathrm{SiO}_{2}$ particles. Talanta 124, 7-13 (2014)

Yen, M.-S., Kuo, M.-C.: Sol-gel synthesis of organic-inorganic hybrid materials comprising boehmite, silica, and thiazole dye. Dyes Pigment. 94, 349-354 (2012)

Zhu, T., Lu, Y.S., Zheng, S.J., Chen, Y.G., Guo, H.B.: Influence of nitric acid acitivation on structure and capacitive performances of ordered mesoporous carbon. Electrochim. Acta 152, 456-463 (2015) 